Homology, Homotopy and Applications, vol.14(2), 2012, pp.63-75

\title{
FORMALITY OF KOSZUL BRACKETS AND DEFORMATIONS OF HOLOMORPHIC POISSON MANIFOLDS
}

\author{
DOMENICO FIORENZA AND MARCO MANETTI
}

(communicated by Jean-Louis Loday)

\begin{abstract}
We show that if a generator of a differential Gerstenhaber algebra satisfies certain Cartan-type identities, then the corresponding Lie bracket is formal. Geometric examples include the shifted de Rham complex of a Poisson manifold and the subcomplex of differential forms on a symplectic manifold vanishing on a Lagrangian submanifold, endowed with the Koszul bracket. As a corollary we generalize a recent result by Hitchin on deformations of holomorphic Poisson manifolds.
\end{abstract}

\section{Dedicated to the memory of J.-L. Loday.}

\section{Introduction}

In the paper [23], Jean-Louis Koszul considered a graded commutative algebra $A=\oplus_{p \in \mathbb{Z}} A^{p}$ with unit $1 \in A^{0}$ and a differential operator $\boldsymbol{l}: A \rightarrow A$ of second order, of odd degree $k$, such that $\boldsymbol{l}(1)=0$ and $\boldsymbol{l}^{2}=0$. Then he proved that the bracket generated by $\boldsymbol{l}$,

$$
[,]_{l}: A^{p} \times A^{q} \rightarrow A^{p+q+k}, \quad[a, b]_{l}:=(-1)^{p}(\boldsymbol{l}(a b)-\boldsymbol{l}(a) b)-a \boldsymbol{l}(b),
$$

satisfies both Poisson and Jacobi identities and then induces on $A$ what is nowadays called a structure of Batalin-Vilkovisky algebra $[\mathbf{6}, \mathbf{1 4}]$.

Koszul's construction applies, in particular, when $A$ is the de Rham complex of a differentiable manifold and $\boldsymbol{l}=\boldsymbol{l}_{\pi}$ is the Lie derivative with respect to a Poisson bivector $\pi$; the degree of $\boldsymbol{l}_{\pi}$ is -1 and then it induces, in particular, a structure of differential graded Lie algebra (DGLA) on the de Rham complex, with degrees shifted by 1 .

However, in this case we have by Cartan formulas that $\boldsymbol{l}_{\pi}=\left[\boldsymbol{i}_{\pi}, d\right]$ and $\left[\boldsymbol{l}_{\pi}, \boldsymbol{i}_{\pi}\right]=0$, where $d$ is the de Rham differential and $\boldsymbol{i}_{\pi}$ is the interior product by $\pi$. As a consequence of this fact, Sharygin and Talalaev [28] obtain that such a differential graded Lie algebra is quasi-isomorphic to an abelian DGLA. In this paper we will reobtain the Sharygin and Talalaev formality theorem as a particular case of a more general

Received May 5, 2012; published on October 14, 2012.

2000 Mathematics Subject Classification: 18G55, 13D10, 53D17.

Key words and phrases: homotopical algebra, differential graded Lie algebra, Batalin-Vilkovisky algebra, deformation theory, Poisson manifold.

Article available at http://intlpress.com/HHA/v14/n2/a4 and doi:10.4310/HHA.2012.v14.n2.a4

Copyright (C) 2012, International Press. Permission to copy for private use granted. 
statement. More precisely, we will show that if $(A, d)$ is a differential graded commutative algebra endowed with a degree $-2 k$ second order differential operator $i: A \rightarrow A$ such that $\boldsymbol{i}(1)=0$, then $(A, d)$ carries a natural Gerstenhaber algebra structure whose underlying DGLA is formal as soon as the differential operator $\boldsymbol{l}=[\boldsymbol{i}, d]$ is such that $[\boldsymbol{l}, \boldsymbol{i}]$ is a second order differential operator. Moreover, our proof also shows that the same conclusion holds for certain subcomplexes of $A$; a remarkable example is the subcomplex of differential forms on a symplectic manifold vanishing on a Lagrangian submanifold.

The formality of the shifted de Rham complex is particularly relevant and useful in formal deformation theory, in view of the fact that quasi-isomorphic DGLAs have isomorphic associated deformation functors. As an application we obtain an extension, and a new proof, of a recent result by Hitchin [18] on deformations of holomorphic Poisson manifolds. Namely, let $\pi$ be a holomorphic Poisson structure on a compact complex manifold $X$, and let $\pi^{\#}: \Omega_{X}^{1} \rightarrow \Theta_{X}$ be the corresponding anchor map. If the natural map $H_{d R}^{2}(X, \mathbb{C}) \rightarrow H^{2}\left(X, \mathcal{O}_{X}\right)$ is surjective, then for every closed $(1,1)$ form $\omega$, the class $\left[\pi^{\#}(\omega)\right] \in H^{1}\left(X, \Theta_{X}\right)$ is tangent to a deformation of $X$ over a smooth basis.

\section{Glossary and background}

We assume that the reader is familiar with the basic theory of differential graded Lie algebras (DGLA), $L_{\infty}$-algebras and related notions (Maurer-Cartan equation, gauge action, etc.). For an introduction to these topics we refer to $[\mathbf{1 2}, \mathbf{1 3}, \mathbf{2 4}, \mathbf{2 6}]$

and the references therein. We shall say that a DGLA is homotopy abelian if it is isomorphic to an abelian DGLA in the homotopy category, i.e., if it is quasiisomorphic to an abelian differential graded Lie algebra.

For the clarity of exposition, we will distinguish the various Lie brackets appearing in this paper according to the following notation:

[,]: The graded commutator bracket, i.e., $[a, b]=a b-(-1)^{\bar{a}} \bar{b} b a$, where $\bar{a}$ is the degree of a homogeneous element $a$;

$[,]_{S N}$ : The Schouten-Nijenhuis bracket on polyvector fields;

$[,]_{\pi}$ : The Koszul bracket associated to a tangent bivector field $\pi$.

Given a graded vector space $V$ we will denote by $V[k]$ the $k$-fold desuspension of $V$, i.e., $V[k]^{i}=V^{k+i}$.

\section{Acknowledgements}

It is a pleasure for us to thank Marco Gualtieri, Donatella Iacono, Rita Pardini, Bruno Vallette and Marco Zambon for useful and interesting discussions on the topics of this paper. We are indebted to Florian Schätz for bringing to our attention the paper $[\mathbf{2 8}]$. 


\section{Review of Koszul brackets}

Let $X$ be a smooth differentiable manifold, $T_{X}$ its tangent bundle and $\left(A_{X}, d\right)=$ $\left(\oplus_{p} \Gamma\left(\bigwedge^{p} T_{X}^{*}\right), d\right)$ its de Rham complex. For every $\eta \in \Gamma\left(\bigwedge^{p} T_{X}\right)$ we denote

$$
\begin{aligned}
& \left.\boldsymbol{i}_{\eta}: A_{X}^{q} \rightarrow A_{X}^{q-p}, \quad \boldsymbol{i}_{\eta}(\alpha)=\eta\right\lrcorner \alpha, \quad \text { the interior product by } \eta, \\
& \boldsymbol{l}_{\eta}: A_{X}^{q} \rightarrow A_{X}^{q-p+1}, \quad \boldsymbol{l}_{\eta}=\left[\boldsymbol{i}_{\eta}, d\right], \quad \text { the Lie derivative. }
\end{aligned}
$$

Recall that for $p=1$, the operator $\boldsymbol{i}_{\eta}$ is a derivation of $A_{X}$ and $\boldsymbol{i}_{\eta \wedge \mu}=\boldsymbol{i}_{\eta} \circ \boldsymbol{i}_{\mu}$.

Everyone is familiar with Cartan's formulas $[\mathbf{5}, \mathbf{1 1}, \mathbf{1 5}]$ :

$$
\left[\boldsymbol{l}_{\eta}, d\right]=0, \quad\left[\boldsymbol{i}_{\eta}, \boldsymbol{i}_{\mu}\right]=0, \quad\left[\boldsymbol{l}_{\eta}, \boldsymbol{i}_{\mu}\right]=\boldsymbol{i}_{[\eta, \mu]_{S N}}, \quad\left[\boldsymbol{l}_{\eta}, \boldsymbol{l}_{\mu}\right]=\boldsymbol{l}_{[\eta, \mu]_{S N}},
$$

where $[,]_{S N}$ is the Schouten-Nijenhuis bracket on polyvector fields.

Definition 2.1 ([23, pg. 266]). The Koszul bracket associated to a tangent bivector field $\pi \in \Gamma\left(\bigwedge^{2} T_{X}\right)$ is the bilinear map $[,]_{\pi}: \bigwedge^{2} A_{X}[1] \rightarrow A_{X}[1]$ defined as

$$
[\alpha, \beta]_{\pi}=(-1)^{p}\left(\boldsymbol{l}_{\pi}(\alpha \wedge \beta)-\boldsymbol{l}_{\pi}(\alpha) \wedge \beta\right)-\alpha \wedge \boldsymbol{l}_{\pi}(\beta), \quad \alpha \in A_{X}^{p}, \beta \in A_{X} .
$$

Using the relation $\boldsymbol{l}_{\pi}=\left[\boldsymbol{i}_{\pi}, d\right]$ we may write, for $\alpha \in A_{X}^{p}, \beta \in A_{X}$,

$$
\begin{aligned}
{[\alpha, \beta]_{\pi}=(-1)^{p}\left(\boldsymbol{i}_{\pi} d(\alpha \wedge \beta)-d \boldsymbol{i}_{\pi}(\alpha \wedge \beta)+d\left(\boldsymbol{i}_{\pi}(\alpha)\right)\right.} & \left.\wedge \beta-\boldsymbol{i}_{\pi}(d \alpha) \wedge \beta\right) \\
& -\alpha \wedge \boldsymbol{i}_{\pi}(d \beta)-\alpha \wedge d\left(\boldsymbol{i}_{\pi}(\beta)\right),
\end{aligned}
$$

and therefore the Koszul bracket of two closed forms is exact.

The restriction of the bracket to $A_{X}^{1}=\Gamma\left(T_{X}^{*}\right)$, also known as the Magri bracket [21, $\mathbf{2 2}$ ], can be conveniently described in terms of the morphism of vector bundles

$$
\pi^{\#}: T_{X}^{*} \rightarrow T_{X},
$$

called the anchor map, defined by the formula

$$
\boldsymbol{i}_{\pi}^{\#(\alpha)}(\beta)=\boldsymbol{i}_{\pi}(\alpha \wedge \beta), \quad \forall \alpha, \beta \in T_{X}^{*} .
$$

In fact it is well known, and in any case easy to prove, that for $\alpha, \beta \in A_{X}^{1}$ we have

$$
\boldsymbol{i}_{\pi \#(\alpha)}(d \beta)=\boldsymbol{i}_{\pi}(\alpha \wedge d \beta)-\alpha \wedge \boldsymbol{i}_{\pi}(d \beta)
$$

and (see, e.g., $[\mathbf{1 5}, \mathbf{2 1}, \mathbf{2 2}, \mathbf{2 9}]$ )

$$
\begin{aligned}
{[\alpha, \beta]_{\pi}=\boldsymbol{l}_{\pi^{\#}(\alpha)}(\beta)-\boldsymbol{l}_{\pi \#(\beta)}(\alpha)-d \boldsymbol{i}_{\pi}(\alpha} & \wedge \beta) \\
& =\boldsymbol{i}_{\pi^{\#}(\alpha)}(d \beta)-\boldsymbol{i}_{\pi \#(\beta)}(d \alpha)+d \boldsymbol{i}_{\pi}(\alpha \wedge \beta) .
\end{aligned}
$$

Assume now that $\pi \in \Gamma\left(\bigwedge^{2} T_{X}\right)$ is a Poisson structure: this means that $[\pi, \pi]_{S N}$ $=0$. By Cartan formulas this implies that

$$
\left[\boldsymbol{l}_{\pi}, \boldsymbol{i}_{\pi}\right]=0, \quad \boldsymbol{l}_{\pi}^{2}=\frac{1}{2}\left[\boldsymbol{l}_{\pi}, \boldsymbol{l}_{\pi}\right]=\frac{1}{2}\left[\left[\boldsymbol{l}_{\pi}, \boldsymbol{i}_{\pi}\right], d\right]=0 .
$$

These conditions ensure (see, e.g., $[\mathbf{4}, \mathbf{2 3}, \mathbf{2 9}]$ and $[\mathbf{1 5}$, Lemma 6.3.4]) that the Koszul bracket satisfies the Jacobi identity, and therefore that the triple $\left(A_{X}, d,[,]_{\pi}\right)$ is a differential Gerstenhaber algebra with an exact generator $\boldsymbol{l}_{\pi}$. These properties will also be reproven in this paper as a byproduct of our computations.

Another important fact, which we will use in Section 6, is that the Poisson structure $\pi$ gives a Lie algebroid structure on the cotangent bundle $T_{X}^{*}[\mathbf{1 5}, \mathbf{2 2}, \mathbf{2 9}$; 
in particular, the anchor map $\pi^{\#}$ is a Lie morphism between the sheaf of 1-forms, endowed with the Koszul-Magri bracket and the sheaf of tangent vector fields [23, equation 3.3].

Having in mind application to deformation theory, in this paper we are mainly interested in the differential graded Lie algebra $\left(A_{X}[1], d,[,]_{\pi}\right)$. In particular, we will be concerned with extensions and generalizations of the following formality theorem:

Theorem 2.2 (Sharygin-Talalaev $[\mathbf{2 8}]$ ). In the notation above, if $\pi$ is a Poisson structure on $X$, then $\left(A_{X}[1], d,[,]_{\pi}\right)$ is a formal differential graded Lie algebra.

We recall that a differential graded Lie algebra is called formal if it is quasiisomorphic to its cohomology. Since the Koszul bracket is trivial in the de Rham cohomology of $X$, the formality of the DGLA $\left(A_{X}[1], d,[,]_{\pi}\right)$ is equivalent to claiming that it is quasi-isomorphic to an abelian differential graded Lie algebra.

We will recover the Sharygin-Talalaev formality theorem in Section 4, as a corollary of a more general statement involving a differential graded commutative algebra $(A, d)$ equipped with an even degree second order differential operator $i: A \rightarrow A$ with $i$ (1) $=0$ and such that $[[\boldsymbol{i}, d], \boldsymbol{i}]$ is also a second order differential operator.

\section{A simple formality criterion for DGLA}

Given a graded vector space $V$ on a characteristic 0 field $\mathbb{K}$, we will denote by $\overline{S(V)}=\oplus_{i \geqslant 1} \bigodot^{i} V$ the graded symmetric coalgebra cogenerated by $V$. Denoting by

$$
D(V)=\operatorname{Hom}_{\mathbb{K}}^{*}(\overline{S(V)}, V)=\prod_{i \geqslant 0} D_{i}(V), \quad \text { where } D_{i}(V)=\operatorname{Hom}_{\mathbb{K}}^{*}\left(\bigodot^{i+1} V, V\right),
$$

the composition on the right with the natural projection $\overline{S(V)} \rightarrow \bigodot^{i+1} V$ gives an inclusion $D_{i}(V) \subset D(V)$, while the composition on the left with the natural projection $\overline{S(V)} \rightarrow V$ gives an isomorphism of graded vector spaces (see, e.g., $[\mathbf{2 0}, \mathbf{2 6}]$ )

$$
\operatorname{Coder}_{\mathbb{K}}^{*}(\overline{S(V)}) \stackrel{\sim}{\rightarrow} D(V) .
$$

By the inverse isomorphism, an element $g$ in $D_{n}(V)$ corresponds to the coderivation

$$
a_{0} \odot a_{1} \odot \cdots \odot a_{n+m} \mapsto \sum_{\sigma} \varepsilon(\sigma) g\left(a_{\sigma(0)}, \ldots, a_{\sigma(m)}\right) \odot a_{\sigma(m+1)} \odot \cdots \odot a_{\sigma(n+m)},
$$

where $\varepsilon(\sigma)$ is the Koszul sign and the sum is carried over all the $(m+1, n)$-unshuffles $\sigma$. The graded vector space $\operatorname{Coder}_{\mathbb{K}}^{*}(\overline{S(V)})$ is a linear subspace of the graded associative algebra $\operatorname{End}_{\mathbb{K}}^{*}(\overline{S(V)})$ of linear endomorphisms of $\overline{S(V)}$, which is closed under the graded commutator bracket; hence $D(V)$ inherits a natural graded Lie algebra structure. A simple computation shows that for $f \in D_{n}(V)$ and $g \in D_{m}(V)$ we have

$$
[f, g]=f \bullet g-(-1)^{\bar{f} \bar{g}} g \bullet f \in D_{n+m}(V),
$$

where

$$
f \bullet g\left(a_{0}, \ldots, a_{n+m}\right)=\sum_{\sigma} \varepsilon(\sigma) f\left(g\left(a_{\sigma(0)}, \ldots, a_{\sigma(m)}\right), a_{\sigma(m+1)}, \ldots, a_{\sigma(n+m)}\right) .
$$

To prevent a possible misinterpretation, let us explicitly remark that the pre-Lie operation $\bullet$ on $D(V)$ is not associative. Notice that the induced bracket on the graded 
Lie subalgebra $D_{0}(V)$ is the same as the graded commutator bracket on $\operatorname{Hom}_{\mathbb{K}}^{*}(V, V)$.

Recall that $L_{\infty}$ structures on the graded vector space $V[-1]$ are the degree 1 elements $\partial$ in $D(V)$ such that $[\partial, \partial]=0$; following $[\mathbf{2 0}]$, an $L_{\infty}$ structure $\partial$ is called linear if $\partial \in D_{0}(V)$.

If $(V, d)$ is a chain complex, then we can look at $(V, d)$ as a linear $L_{\infty}$-algebra, and so at $d$ as an $L_{\infty}$ structure on $V$. Using $d$ (seen as a coderivation) to "translate the origin" in $\operatorname{Coder}_{\mathbb{K}}^{*}(\overline{S(V)})$, we have that $L_{\infty}$ structures on $V$ can be seen as the degree 1 coderivations $\xi$ on $\overline{S(V)}$ such that $(d+\xi)^{2}=0$. This is conveniently rewritten as the Maurer-Cartan equation for the DGLA $\operatorname{Coder}_{\mathbb{K}}^{*}(\overline{S(V)})$ :

$$
\delta \xi+\frac{1}{2}[\xi, \xi]=0
$$

where $\delta$ is the adjoint of $d$ seen as a coderivation.

For any degree zero coderivation $R \in D_{>0}(V)=\prod_{i>0} D_{i}(V)$, the exponential $e^{R}$ is a well defined element in the graded associative algebra $\operatorname{End}_{\mathbb{K}}^{*}(\overline{S(V)})$, and it is immediate to see that, since $R$ is a coderivation, $e^{R}$ is actually a graded coalgebra automorphism of $\overline{S(V)}$ with inverse $e^{-R}$. Moreover, in the graded associative algebra $\operatorname{End}_{\mathbb{K}}^{*}(\overline{S(V)})$ we have, for any solution $\xi$ of the Maurer-Cartan equation in $D(V)$,

$$
e^{R}(d+\xi) e^{-R}=d+e^{R} * \xi
$$

where $*$ denotes the gauge action in $D(V)$ (see, e.g., $[\mathbf{2 7}]$ ):

$$
e^{R} * \xi=\xi+\sum_{n=0}^{\infty} \frac{\left(\operatorname{ad}_{R}\right)^{n}}{(n+1) !}([R, \xi]+[R, d]) .
$$

In particular, for any degree zero coderivation $R \in D_{>0}(V)$, the coderivation $\xi_{R}=$ $e^{R} * 0$ defines an $L_{\infty}$-algebra structure isomorphic (via $e^{R}$ ) to a linear one: $e^{R} d e^{-R}=$ $d+\xi_{R}$.

Remark 3.1. The isomorphism $e^{R}$ can be conveniently written in terms of an operadic "forest formula". Namely, the $\operatorname{Hom}_{\mathbb{K}}\left(\bigodot^{m} V, \bigodot^{n} V\right)$-component of $e^{R}$ can be written as a weighted sum over oriented forests with $n$ roots and $m$ leaves, and whose internal $k$-valent vertices are decorated by the $\operatorname{Hom}_{\mathbb{K}}\left(\bigodot^{k} V, V\right)$-component of $R$. As usual in this kind of formula, the weights are given by the (inverse of the) cardinality of the automorphism groups of the forests.

Theorem 3.2. Let $(A, d)$ be a chain complex, let $R \in \operatorname{Hom}_{\mathbb{K}}^{-2 k}(A \odot A, A)$ be considered as a degree zero element of $D(A[2 k])$, let $Q=[R, d]$, and let

$$
[a, b]_{Q}=(-1)^{\bar{a}} Q(a, b)
$$

be the degree zero bracket on $A[2 k-1]$ induced by $Q$ via decalage. If $[R, Q]=0$, then the bracket (1) gives a formal homotopy abelian DGLA structure on $(A[2 k-1], d)$. More precisely, the exponential of the coderivation $R$ is an $L_{\infty}$-isomorphism between the DGLA $(A[2 k-1], d, 0)$ and $\left(A[2 k-1], d,[,]_{Q}\right)$.

Proof. Since $R$ is a degree zero element of $D_{>0}(A[2 k])$ and $[R,[R, d]]=0$, we have

$$
e^{R} d e^{-R}=d+e^{R} * 0=d+\sum_{n=0}^{\infty} \frac{\left(\operatorname{ad}_{R}\right)^{n}}{(n+1) !}([R, d])=d+Q .
$$

Hence the two DGLAs $(A[2 k-1], d, 0)$ and $\left(A[2 k-1], d,[,]_{Q}\right)$ have isomorphic Bar 
constructions, i.e., they are isomorphic as $L_{\infty}$-algebras. Therefore, according to the Bar-Cobar resolution [24], they are quasi-isomorphic as differential graded Lie algebras.

The above theorem is one of the possible formality criteria and finds application only in some particular cases, for instance for the Koszul brackets. The reader may find similar results in $[\mathbf{9}, \mathbf{1 6}]$ and $[\mathbf{2 6}$, Thm. 9.13].

\section{Differential operators on graded commutative algebras}

The theory of differential operators on commutative rings (see, e.g., $[\mathbf{8}, \mathbf{1 5}]$ ) extends without difficulties to the graded case. Let $A=\oplus A^{i}$ be a graded commutative algebra with unit $1 \in A^{0}$ over a field $\mathbb{K}$ of characteristic 0 . Every $a \in A$ is also considered as an element of $\operatorname{Hom}_{\mathbb{K}}^{*}(A, A)$ acting by left multiplication:

$$
a: A \rightarrow A, \quad a(b)=a b .
$$

Denote by [,] the graded commutator on $\operatorname{Hom}_{\mathbb{K}}^{*}(A, A)$ and by

$$
\operatorname{Diff}_{k}(A)=\bigoplus_{n \in \mathbb{Z}} \operatorname{Diff}_{k}^{n}(A) \subset \operatorname{Hom}_{\mathbb{K}}^{*}(A, A)
$$

the graded subspace of differential operators of order $\leqslant k$. Recall that $\operatorname{Diff}_{k}(A)$ is defined recursively by setting $\operatorname{Diff}_{k}(A)=0$ for $k<0$ and

$$
\operatorname{Diff}_{k}(A)=\left\{f \in \operatorname{Hom}_{\mathbb{K}}^{*}(A, A) \mid[f, a] \in \operatorname{Diff}_{k-1}(A) \forall a \in A\right\}
$$

for $k \geqslant 0$.

Moreover,

$$
\operatorname{Diff}_{k}(A) \operatorname{Diff}_{h}(A) \subset \operatorname{Diff}_{h+k}(A), \quad\left[\operatorname{Diff}_{k}(A), \operatorname{Diff}_{h}(A)\right] \subset \operatorname{Diff}_{h+k-1}(A),
$$

and therefore the space $\operatorname{Diff}(A)=\bigcup_{k} \operatorname{Diff}_{k}(A)$ of differential operators is a Lie subalgebra of $\operatorname{Hom}_{\mathbb{K}}^{*}(A, A)$.

The differential operators of order $\leqslant k$ are stable under scalar extension: if $f \in$ $\operatorname{Diff}_{k}(A)$ and $B$ is a graded commutative algebra, then $f \otimes I d \in \operatorname{Diff}_{k}\left(A \otimes_{\mathbb{K}} B\right)$.

For a fixed even integer $2 k$, let $V=A[2 k]$, i.e., $V=\oplus_{i \in \mathbb{Z}} V^{i}$ with $V^{i}=A^{i+2 k}$. According to the natural isomorphism $D_{0}(V)=\operatorname{Hom}_{\mathbb{K}}^{*}(V, V)=\operatorname{Hom}_{\mathbb{K}}^{*}(A, A)$, we may consider Diff $(A)$ as a Lie subalgebra of $D(V)$.

Also, for every $n \geqslant 0$ consider the multiplication map

$$
\mu_{n}: A^{\odot n+1} \rightarrow A, \quad \mu_{n}\left(a_{0} \odot \cdots \odot a_{n}\right)=a_{0} a_{1} \cdots a_{n} .
$$

We shall look at $\mu_{n}$ as a degree $2 k n$ element in $D_{n}(V)$, for every $n \geqslant 0$.

Remark 4.1. The Lie subalgebra of $D(A)$ generated by the operators $\mu_{n}, n \geqslant 0$ is isomorphic to the Lie algebra of polynomial vector fields on the affine line vanishing in the origin, with $\mu_{n}$ corresponding to $\frac{-t^{n+1}}{(n+1) !} \frac{d}{d t}$.

Lemma 4.2. For a linear map $f \in \operatorname{Hom}_{\mathbb{K}}^{*}(A, A)$ the following conditions are equivalent:

1. $f \in \operatorname{Der}_{\mathbb{K}}^{*}(A)$; 
2. $\left[f, \mu_{n}\right]=0$ for every $n>0$;

3. $\left[f, \mu_{1}\right]=0$.

Proof. For every $a, b \in A$ we have

$$
\left[f, \mu_{1}\right](a, b)=f(a b)-f(a) b-(-1)^{\bar{a} \bar{b}} f(b) a=f(a b)-f(a) b-(-1)^{\bar{a}} \bar{f} a f(b),
$$

and therefore $f$ is a derivation if and only if $\left[f, \mu_{1}\right]=0$. The proof that if $f$ is a derivation then $\left[f, \mu_{n}\right]=0$ for every $n>0$ is easy and omitted.

Theorem 4.3. For a linear map $f \in \operatorname{Hom}_{\mathbb{K}}^{*}(A, A)$, the following conditions are equivalent:

1. $f \in \operatorname{Diff}_{2}(A)$ and $f(1)=0$;

2. $f$ satisfies the "seven terms" condition

$$
\begin{aligned}
f(a b c)+f(a) b c+(-1)^{\bar{a} \bar{b}} f(b) a c+ & (-1)^{\bar{c}(\bar{a}+\bar{b})} f(c) a b \\
& =f(a b) c+(-1)^{\bar{a}(\bar{b}+\bar{c})} f(b c) a+(-1)^{\bar{b} \bar{c}} f(a c) b ;
\end{aligned}
$$

3. The bilinear form $\Phi(a, b)=f(a b)-f(a) b-(-1)^{\bar{a} \bar{f}}$ af $(b)$ satisfies the Poisson identity

$$
\Phi(a, b c)=\Phi(a, b) c+(-1)^{(\bar{a}+\bar{f}) \bar{b}} b \Phi(a, c) ;
$$

4. $\left[f, \mu_{2}\right]=\left[\left[f, \mu_{1}\right], \mu_{1}\right]$.

Proof. If $f \in \operatorname{Diff}_{2}(A)$ then $[[[f, a], b], c]=0$ for every $a, b, c \in A$ and if, in addition, $f(1)=0$ then also

$$
[[[f, a], b], c](1)+f(1) a b c=0
$$

for every $a, b, c \in A$. Expanding the above expression one finds the seven terms condition, hence (1) implies (2). That (2) implies (3) is immediate. Next, the Poisson identity means that for every $a$ the operator $\Phi(a,-)$ is a derivation. Since $[f, a]=$ $\Phi(a,-)+f(a)$, this implies that $[f, a] \in \operatorname{Diff}_{1}(A)$ for any $a$, and so $f \in \operatorname{Diff}_{2}(A)$. Moreover, by the Poisson identity again, $f(1)=-\Phi(1,1)=0$. This shows that (3) implies (1). Finally, showing that (4) is equivalent to (2) is tedious but straightforward.

Definition 4.4. A linear map $f: A \rightarrow A$ will be called a quasi-Batalin-Vilkovisky operator if it satisfies any of the equivalent conditions of Theorem 4.3.

Remark 4.5. The name quasi-Batalin-Vilkovisky operator is motivated from the fact $[\mathbf{1 4}, \mathbf{2 3}]$ that a Batalin-Vilkovisky algebra may be defined as the data of a graded commutative algebra $A$ and a quasi-BV operator $\Delta$ of odd degree such that $\Delta^{2}=0$.

\section{Formality of Koszul brackets}

Throughout this section, $(A, d)$ will be a differential graded commutative algebra (with a differential $d$ of degree 1) over a field of characteristic 0, and $i$ a quasi-BV operator on $A$ of even degree $-2 k$. We will write $\boldsymbol{l}=[\boldsymbol{i}, d]$. Since $d$ is a derivation, $\boldsymbol{l}$ is also a quasi-BV operator on $A$ of degree $-2 k+1$. 
Lemma 5.1. In the notation above, assume that $[\boldsymbol{l}, \boldsymbol{i}]$ is also a quasi-BV operator on A. Let $R \in \operatorname{Hom}_{\mathbb{K}}^{-2 k}(A \odot A, A)$ and $Q \in \operatorname{Hom}_{\mathbb{K}}^{-2 k+1}(A \odot A, A)$ be the bilinear operators defined respectively as

$$
R(a, b)=\boldsymbol{i}(a b)-\boldsymbol{i}(a) b-a \boldsymbol{i}(b)
$$

and

$$
Q(a, b)=\boldsymbol{l}(a b)-\boldsymbol{l}(a) b-(-1)^{\bar{a}} a \boldsymbol{l}(b) .
$$

Then $Q=[R, d]$ and $[Q, R]=0$ in the graded Lie algebra $D(A[2 k])$.

Proof. It is immediate to check that in $D(A[2 k])$ one has $Q=[R, d]$. Moreover, by definition, $R=\left[\boldsymbol{i}, \mu_{1}\right]$ and $Q=\left[\boldsymbol{l}, \mu_{1}\right]$. By assumption we have

$$
\left[\boldsymbol{i}, \mu_{2}\right]=\left[\left[\boldsymbol{i}, \mu_{1}\right], \mu_{1}\right], \quad\left[\boldsymbol{l}, \mu_{2}\right]=\left[\left[\boldsymbol{l}, \mu_{1}\right], \mu_{1}\right], \quad\left[[\boldsymbol{l}, \boldsymbol{i}] \mu_{2}\right]=\left[\left[[\boldsymbol{l}, \boldsymbol{i}] \mu_{1}\right], \mu_{1}\right] .
$$

The graded Jacobi identity gives

$$
\begin{aligned}
{\left[\left[[\boldsymbol{l}, \boldsymbol{i}], \mu_{1}\right], \mu_{1}\right] } & =\left[\left[\left[\boldsymbol{l}, \mu_{1}\right], \boldsymbol{i}\right], \mu_{1}\right]+\left[\left[\boldsymbol{l},\left[\boldsymbol{i}, \mu_{1}\right]\right], \mu_{1}\right] \\
& =\left[\left[\left[\boldsymbol{l}, \mu_{1}\right], \mu_{1}\right], \boldsymbol{i}\right]+2\left[\left[\boldsymbol{l}, \mu_{1}\right],\left[\boldsymbol{i}, \mu_{1}\right]\right]+\left[\boldsymbol{l},\left[\left[\boldsymbol{i}, \mu_{1}\right], \mu_{1}\right]\right]
\end{aligned}
$$

and

$$
\left[[\boldsymbol{l}, \boldsymbol{i}], \mu_{2}\right]=\left[\left[\boldsymbol{l}, \mu_{2}\right], \boldsymbol{i}\right]+\left[\boldsymbol{l},\left[\boldsymbol{i}, \mu_{2}\right]\right]
$$

Therefore,

$$
0=\left[\left[[\boldsymbol{l}, \boldsymbol{i}], \mu_{1}\right], \mu_{1}\right]-\left[[\boldsymbol{l}, \boldsymbol{i}], \mu_{2}\right]=2\left[\left[\boldsymbol{l}, \mu_{1}\right],\left[\boldsymbol{i}, \mu_{1}\right]\right]=2[Q, R] .
$$

Example 5.2. Let $\left(A_{X}, d\right)$ be the de Rham complex of a manifold $X$. Given $\eta \in$ $\Gamma\left(\bigwedge^{p} T_{X}\right)$ we have $\boldsymbol{i}_{\eta} \in \operatorname{Diff}_{p}\left(A_{X}\right)$; moreover, $\boldsymbol{i}_{\eta} \in \operatorname{Diff}_{p-1}\left(A_{X}\right)$ if and only if $\eta=0$. According to the formula

$$
\left[\boldsymbol{l}_{\eta}, \boldsymbol{i}_{\beta}\right]=\boldsymbol{i}_{[\eta, \beta]_{S N}},
$$

we have that, for $\pi \in \Gamma\left(\bigwedge^{2} T_{X}\right)$, the operators $\boldsymbol{i}_{\pi}$ and $\left[\boldsymbol{l}_{\pi}, \boldsymbol{i}_{\pi}\right]$ are quasi-BV if and only if $[\pi, \pi]_{S N}=0$, i.e., if and only if $\pi$ is a Poisson structure.

From Theorem 3.2 we therefore obtain

Theorem 5.3. In the notation above, assume that $[\boldsymbol{l}, \boldsymbol{i}]$ is also a quasi-BV operator on $A$, and let $[,]_{l}$ be the degree $-2 k+1$ bracket on $A$ defined by

$$
[a, b]_{\boldsymbol{l}}=(-1)^{\bar{a}}(\boldsymbol{l}(a b)-\boldsymbol{l}(a) b)-a \boldsymbol{l}(b) .
$$

Then $\left(A, d, \cdot,[,]_{l}\right)$ is a Gerstenhaber algebra, whose underlying DGLA $(A[2 k-1], d$, $\left.[,]_{l}\right)$ is a homotopy abelian DGLA. If, in addition, $\boldsymbol{l}^{2}=0$, then $\left(A, d, \cdot,[,]_{\boldsymbol{l}}, \boldsymbol{l}\right)$ is a Batalin-Vilkovisky algebra.

Proof. The only thing to be checked is the Poisson identity for the bracket $[,]_{l}$; by Theorem 4.3, this is equivalent to saying that $\boldsymbol{l}$ is a quasi-BV operator.

Remark 5.4. An alternative proof of the above theorem can be given using the results of [2], where it is (implicitly) proved that the series of higher Koszul brackets gives a morphism of graded Lie algebras and then commutes with adjoint actions; this is essentially the argument used in [7]. 
Example 5.5. An immediate application of the above theorem is the following refined version of Theorem 2.2: Let $X$ be a smooth manifold, $\left(A_{X}, d, \wedge\right)$ be its de Rham algebra, and let $[,]_{\pi}$ be the Koszul bracket induced by a Poisson bivector field $\pi$. By Example 5.2, the operator $\boldsymbol{i}_{\pi}$ satisfies the hypothesis of Theorem 5.3, and so $\left(A_{X}, d, \wedge,[,]_{\pi}\right)$ is a Gerstenhaber algebra whose underlying DGLA $\left(A_{X}[1], d,[,]_{\pi}\right)$ is homotopy abelian.

Corollary 5.6. In the hypothesis of Theorem 5.3, let $B$ a differential graded linear subspace of $A$ which is closed under the bilinear operator $R=\left[\boldsymbol{i}, \mu_{1}\right]$. Then $\left(B[2 k-1], d,[,]_{l}\right)$ is a formal DGLA.

Proof. Since $B$ is closed under $R$, then $\overline{S(B)}$ is preserved by $e^{R}$ and the proof of Theorem 3.2 applies.

Example 5.7. Let $A$ and $\boldsymbol{i}$ be as in Theorem 5.3. Then, for any $p_{0} \geqslant 2 k$, the subcomplex $B=\bigoplus_{p \geqslant p_{0}} A^{p}$ satisfies the assumptions of Corollary 5.6.

Example 5.8. Let $X$ be a symplectic manifold, and let $j: Y \hookrightarrow X$ be the inclusion of a Lagrangian submanifold. Then the differential ideal $B:=\operatorname{ker} j^{*} \subset A_{X}$ satisfies the assumptions of Corollary 5.6; this immediately follows by the Lagrangian Neighborhood Theorem. In particular, the Koszul bracket induces a homotopy abelian DGLA structure on the (shifted) complex of differential forms vanishing on $Y$.

Corollary 5.9. Let $A_{\bullet}$ be a cosimplicial commutative differential graded algebra, and let $\boldsymbol{i}_{\bullet}: A_{\bullet} \rightarrow A_{\bullet}$ be a cosimplicial linear map such that $\boldsymbol{i}_{n}: A_{n} \rightarrow A_{n}$ satisfies the assumption of Theorem 5.3 for every $n$. Then the totalization of the cosimplicial $\operatorname{DGLA}\left(A_{\bullet}[2 k-1], d_{\bullet},[,]_{\boldsymbol{\bullet}_{\bullet}}\right)$ is a homotopy abelian differential graded Lie algebra.

Proof. The proof is an immediate consequence of the definition of totalization, see, e.g., $[\mathbf{3}, \mathbf{1 0}, \mathbf{1 2}, \mathbf{1 7}, \mathbf{1 9}]$, and of the fact that differential operators of order $\leqslant 2$ are stable under scalar extension.

\section{An application to deformations of holomorphic Poisson manifolds}

In this section we will denote by $X$ a compact complex manifold, by $\Theta_{X}$ and $\Omega_{X}^{1}$ the sheaves of holomorphic vector fields and holomorphic 1-forms respectively, by $A_{X}^{p, q}$ the space of differentiable forms of type $(p, q)$ and by $H_{d R}^{*}(X, \mathbb{C})$ the de Rham cohomology of $X$.

A holomorphic Poisson structure on a complex manifold $X$ is a holomorphic tangent bivector field $\pi \in H^{0}\left(X, \bigwedge^{2} \Theta_{X}\right)$ such that $[\pi, \pi]_{S N}=0$. As in the differentiable case, the Poisson structure induces both a Koszul bracket

$$
[,]_{\pi}: A_{X}^{p, q} \times A_{X}^{r, s} \rightarrow A_{X}^{p+r-1, q+s}
$$

and an anchor map $\pi^{\#}: \Omega_{X}^{1} \rightarrow \Theta_{X}$ which is a morphism of sheaves of Lie algebras.

Denoting by $F_{X}^{0} \supset F_{X}^{1} \supset \cdots$ the Hodge filtration,

$$
F_{X}^{i}=\bigoplus_{p \geqslant i, q} A_{X}^{p, q}
$$

we have, by previous results, that the DGLA $\left(F_{X}^{0}[1], d,[,]_{\pi}\right)$ is quasi-isomorphic to 
an abelian DGLA, and $\left(F_{X}^{1}[1], d,[,]_{\pi}\right)$ is a differential graded Lie subalgebra. This is not sufficient to ensure the formality of $F_{X}^{1}[1]$. However, we have

Lemma 6.1. Assume that the inclusion $F_{X}^{1} \hookrightarrow F_{X}^{0}$ is injective in cohomology (e.g., if $X$ is Kähler); then the DGLA $\left(F_{X}^{1}[1], d,[,]_{\pi}\right)$ is quasi-isomorphic to an abelian $D G L A$.

Proof. This is an easy consequence of the homotopy classification of DGLAs and $L_{\infty}$-algebras [20]. Indeed, let $f: \mathfrak{g} \rightarrow \mathfrak{h}$ be a DGLA morphism, with $H^{*}(f)$ injective and $\mathfrak{h}$ quasi-abelian. Then, by the fact that $\mathfrak{h}$ quasi-abelian, we have a zigzag of quasiisomorphisms of DGLAs $\mathfrak{h} \stackrel{\sim}{\leftarrow} \stackrel{\sim}{\longrightarrow} V$ with $V$ a graded vector space (considered as a DGLA with trivial differential and bracket). Let the DGLA $\mathfrak{l}$ be the homotopy fiber product of $\mathfrak{g}$ with $\mathfrak{k}$ over $\mathfrak{h}$, let the graded vector space $W$ be the image of $H^{*}(\mathfrak{l}) \rightarrow H^{*}(\mathfrak{k}) \cong H^{*}(V)=V$, and let $\pi_{W}: V \rightarrow W$ be a graded linear projection. Then we have a homotopy commutative diagram of DGLAs

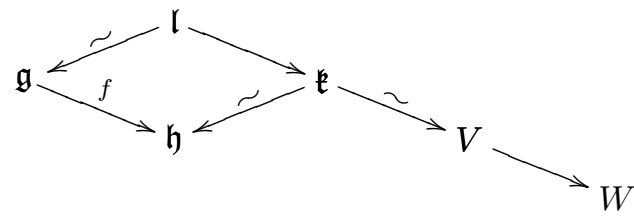

whose homotopy commutative square on the left is a homotopy pullback. In particular, the morphism $\mathfrak{l} \rightarrow \mathfrak{g}$ is a quasi-isomorphism, and so the morphism $\mathfrak{l} \rightarrow \mathfrak{k}$ is injective in cohomology. Therefore, the composition $\mathfrak{l} \rightarrow W$ is a quasi-isomorphism and we have the zigzag of quasi-isomorphisms $\mathfrak{g} \stackrel{\sim}{\leftarrow} \stackrel{\sim}{\longrightarrow} W$, with $W$ abelian.

If we are interested in obstructions of lifting Maurer-Cartan elements, then the assumption of Lemma 6.1 can be relaxed. Denoting by Art the category of local Artinian $\mathbb{C}$-algebras, for any DGLA $L$ the associated deformation functor $\operatorname{Def}_{L}$ : Art $\rightarrow$ Set is defined as

$$
\operatorname{Def}_{L}(C)=\frac{\left\{x \in L^{1} \otimes \mathfrak{m}_{C} \mid d x+\frac{1}{2}[x, x]=0\right\}}{\text { gauge equivalence }},
$$

where $\mathfrak{m}_{C}$ is the maximal ideal of $C$. Among the basic facts about DGLA and associated deformation functors we have (see, e.g., $[\mathbf{2 6}, \mathbf{2 7}]$ for proofs and more details):

1. Quasi-isomorphic DGLAs have isomorphic associated deformation functors;

2. Abelian DGLAs have unobstructed associated deformation functors;

3. If $L \rightarrow M$ is a morphism of DGLAs and $\operatorname{Def}_{M}$ is unobstructed, then the obstructions of $\operatorname{Def}_{L}$ are contained in the kernel of $H^{2}(L) \rightarrow H^{2}(M)$.

Lemma 6.2. Assume that the natural map $H_{d R}^{2}(X, \mathbb{C}) \rightarrow H^{2}\left(X, \mathcal{O}_{X}\right)$ is surjective. Then the functor $\operatorname{Def}_{F_{X}^{1}[1]}$ is unobstructed.

Proof. Since $F_{X}^{0}[1]$ is quasi-isomorphic to an abelian DGLA, the functor $\operatorname{Def}_{F_{X}^{0}[1]}$ is unobstructed, and therefore the obstructions of $\operatorname{Def}_{F_{X}^{1}[1]}$ are contained in the kernel 
of $H^{2}\left(F_{X}^{1}[1]\right) \rightarrow H^{2}\left(F_{X}^{0}[1]\right)$. Now the exact sequence

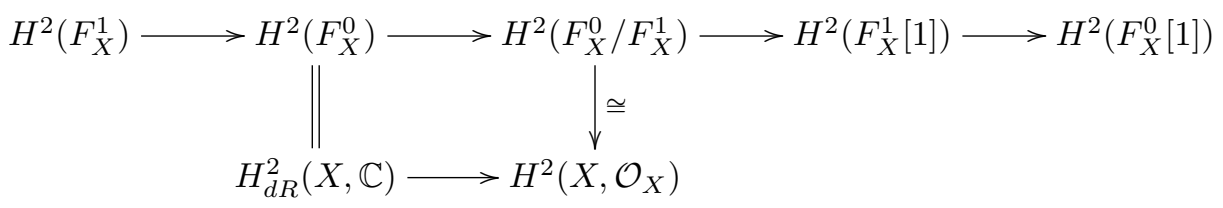

implies that $H^{2}\left(F_{X}^{1}[1]\right) \rightarrow H^{2}\left(F_{X}^{0}[1]\right)$ is injective.

Theorem 6.3. Let $\pi$ be a holomorphic Poisson structure on a compact complex manifold $X$ such that the natural map $H_{d R}^{2}(X, \mathbb{C}) \rightarrow H^{2}\left(X, \mathcal{O}_{X}\right)$ is surjective. Then for every closed $(1,1)$ form $\omega$, the class $\left[\pi^{\#}(\omega)\right] \in H^{1}\left(X, \Theta_{X}\right)$ is tangent to a deformation of $X$ over a smooth basis.

Proof. Since $X$ is compact, it has a semiuniversal deformation. According to Artin's theorem on the solution of analytic equations [1], it is sufficient to prove that the class of $\pi^{\#}(\omega)$ extends to a formal deformation over $\mathbb{C}[[t]]$. The anchor map $\pi^{\#}$, being holomorphic, extends to a morphism of differential graded Lie algebras

$$
\gamma: F_{X}^{1}[1] \stackrel{\text { projection }}{\longrightarrow} A_{X}^{0, *}\left(\Omega_{X}^{1}\right) \stackrel{\pi^{\#}}{\longrightarrow} A_{X}^{0, *}\left(\Theta_{X}\right) .
$$

The DGLA $A_{X}^{0, *}\left(\Theta_{X}\right)$ is the Kodaira-Spencer algebra of $X$, and its associated deformation functor is isomorphic to the functor of infinitesimal deformations of $X$; see [25] and the references therein. According to Lemma 6.2 the functor $\operatorname{Def}_{F_{X}^{1}[1]}$ is unobstructed, and therefore the class

$$
[\omega] \in H^{1}\left(F_{X}^{1}[1]\right) \cong \operatorname{Def}_{F_{X}^{1}[1]}\left(\frac{\mathbb{C}[t]}{\left(t^{2}\right)}\right)
$$

extends to an element of $\operatorname{Def}_{F_{X}^{1}[1]}(\mathbb{C}[[t]])$. This implies, in particular, that $\gamma([\omega])=$ $\left[\pi^{\#}(\omega)\right]$ extends to a deformation of $X$ over $\mathbb{C}[[t]]$.

Remark 6.4. Theorem 6.3 has been recently proved by Hitchin [18] under the assumption that either $X$ is Kähler or $H^{2}\left(X, \mathcal{O}_{X}\right)=0$. The proof of Theorem 6.3 also shows that the assumption $\partial \omega=\bar{\partial} \omega=0$ can be replaced by the existence of a form $\eta \in A_{X}^{2,0}$ such that $\partial \eta=0, \bar{\partial} \eta=\partial \omega$ and $\bar{\partial} \omega=0$. In fact, since $\gamma(\omega)=\gamma(\omega-\eta)$, it is sufficient to consider the cohomology class $[\omega-\eta] \in H^{1}\left(F_{X}^{1}[1]\right)$ as a Maurer-Cartan element.

\section{References}

[1] M. Artin, On the solutions of analytic equations, Invent. Math. 5 (1968), no. 4, $277-291$.

[2] K. Bering, P.H. Damgaard and J. Alfaro, Algebra of higher antibrackets, Nuclear Phys. B 478 (1996), no. 1-2, 459-503.

[3] P. Bressler, A. Gorokhovsky, R. Nest and B. Tsygan, Deformations of algebroid stacks, Adv. Math. 226 (2011), no. 4, 3018-3087.

[4] J.-L. Brylinski, A differential complex for Poisson manifolds, J. Differential Geom. 28 (1988), no. 1, 93-114. 
[5] H. Cartan, Notion d'algèbre différentielle; applications aux groupes de Lie et aux variétés où opère un groupe de Lie, Colloque de Topologie, tenu à Bruxelles, 5-8 juin 1950, CBRM, Georges Thone, Liège (1950), 15-27.

[6] A. Cattaneo, D. Fiorenza and R. Longoni, Graded Poisson algebras, Encyclopedia of Mathematical Physics (J.-P. Naber and G. Sheung Tsun Tsou, Eds.), Academic Press, New York (2006), 560-567.

[7] A. Cattaneo and F. Schätz, Equivalence of higher derived brackets, J. Pure Appl. Algebra 212 (2008), no. 11, 2450-2460.

[8] S.C. Coutinho, A primer of algebraic D-modules, Cambridge Univ. Press, Cambridge (1995).

[9] V. Dotsenko, S. Shadrin and B. Vallette, De Rham cohomology and homotopy Frobenius manifolds, arXiv:math.KT/1203.5077v2.

[10] D. Fiorenza and M. Manetti, $L_{\infty}$ structures on mapping cones, Algebra Number Theory 1 (2007), no. 3, 301-330.

[11] D. Fiorenza and M. Manetti, A period map for generalized deformations, $J$. Noncommut. Geom. 3 (2009), no. 4, 579-597.

[12] D. Fiorenza, M. Manetti and E. Martinengo, Cosimplicial DGLAs in deformation theory, Comm. Algebra 40 (2012), no. 6, 2243-2260.

[13] Y. Frégier and M. Zambon, $L_{\infty}$-algebras governing simultaneous deformations via derived brackets, arXiv:math.QA/1202.2896v1.

[14] E. Getzler, Batalin-Vilkovisky algebras and two-dimensional topological field theories, Comm. Math. Phys. 159 (1994), no. 2, 265-285.

[15] V. Ginzburg, Lectures on noncommutative geometry, arXiv:math.AG/ $0506603 \mathrm{v} 1$.

[16] D. Grandini, Y.S. Poon and B. Rolle, Differential Gerstenhaber algebras of generalized complex structures, arXiv:math.DG/1109.3966v1.

[17] V. Hinich, Descent of Deligne groupoids, Internat. Math. Res. Notices (1997), no. $5,223-239$.

[18] N. Hitchin, Deformations of holomorphic Poisson manifolds, arXiv:math.DG/ $1105.4775 \mathrm{v} 1$.

[19] D. Iacono and M. Manetti, An algebraic proof of Bogomolov-Tian- Todorov theorem, in Deformation spaces (H. Abbaspour, M. Marcolli and T. Tradler, Eds.), Vieweg + Teubner Verlag, Wiesbaden (2010), 113-133.

[20] M. Kontsevich, Deformation quantization of Poisson manifolds, I, Letters in Mathematical Physics 66 (2003), no. 3, 157-216.

[21] Y. Kosmann-Schwarzbach and F. Magri, Poisson-Nijenhuis structures, Ann. Inst. Henri Poincaré 53 (1990), no. 1, 35-81.

[22] Y. Kosmann-Schwarzbach, Poisson manifolds, Lie algebroids, modular classes: a survey, SIGMA 4 (2008), 005, 30 pages.

[23] J.-L. Koszul, Crochet de Schouten-Nijenhuis et cohomologie, in Astérisque (Numéro Hors Série) (1985), 257-271.

[24] J.-L. Loday and B. Vallette, Algebraic operads, Grundlehren der mathematischen Wissenschaften 346, Springer-Verlag, New York (2012). 
[25] M. Manetti, Cohomological constraint to deformations of compact Kähler manifolds, Adv. Math. 186 (2004), no. 1, 125-142.

[26] M. Manetti, Lectures on deformations of complex manifolds, Rend. Mat. Appl. (7) 24 (2004), no. 1, 1-183.

[27] M. Manetti, Differential graded Lie algebras and formal deformation theory (Algebraic Geometry-Seattle 2005), Proc. Sympos. Pure Math. 80 (2009), 785810, Amer. Math. Soc., Providence, RI.

[28] G. Sharygin and D. Talalaev, On the Lie-formality of Poisson manifolds, $J$. K-Theory 2 (2008), no. 2, Special issue in memory of Yurii Petrovich Solovyev, Part 1, 361-384.

[29] P. Xu, Gerstenhaber algebras and BV-algebras in Poisson geometry, Comm. Math. Phys. 200 (1999), no. 3, 545-560.

Domenico Fiorenza fiorenza@mat.uniroma1.it

Marco Manetti manetti@mat.uniroma1.it

Università degli Studi di Roma "La Sapienza", Dipartimento di Matematica "Guido Castelnuovo", P.le Aldo Moro 5, I-00185 Roma, Italy 